\title{
Prognostic Evaluation Between Apache II Scale and Ranson Scale in Severe Acute Pancreatitis in the Emergency Department
}

ISSN: 2637-7632

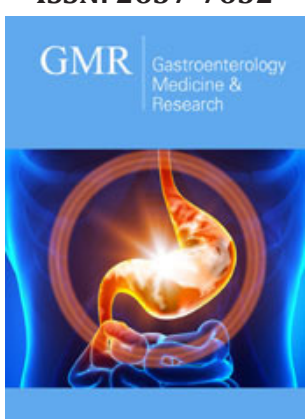

*Corresponding author: Miguel Ángel Pedraza Zárate, Coordination of $\mathrm{Nu}$ trition and Dietetics, OOAD Veracruz Norte, Mexican Social Security Institute, Xalapa, Veracruz, Mexico

Submission: 滥 December 17, 2020

Published: March 29, 2021

Volume 5 - Issue 5

How to cite this article: Juan Carlos MezaFranco, Miguel Ángel Pedraza-Zárate, Juan Pablo Solis-Pureco, Dimas Muñoz-Flores, Jose Luis Garcia-Pérez et al. Prognostic Evaluation Between Apache II Scale and Ranson Scale in Severe Acute Pancreatitis in the Emergency Department. Gastro Med Res. 5(5). GMR. 000622. 2021.

DOI: 10.31031/GMR.2021.05.000622

Copyright@ Miguel Ángel PedrazaZárate, This article is distributed under the terms of the Creative Commons Attribution 4.0 International License, which permits unrestricted use and redistribution provided that the original author and source are credited.
Juan Carlos Meza-Franco ${ }^{1}$, Miguel Ángel Pedraza-Zárate ${ }^{2 *}$, Juan Pablo SolisPureco $^{1}$, Dimas Muñoz-Flores ${ }^{1}$, Jose Luis Garcia-Pérez ${ }^{1}$, Anahari UscangaMontesano $^{3}$, Sile Araceli Ortiz Zavaleta and Edith Guillen Salomon ${ }^{4}$

${ }^{1}$ Emergency Service, UMAE Hospital de Especialidades \# 14 CMN Adolfo Ruiz Cortines, IMSS, Veracruz, Ver, Mexico

${ }^{2}$ Coordination of Nutrition and Dietetics, OOAD Veracruz Norte, Mexican Social Security Institute, Xalapa, Veracruz, Mexico

${ }^{3}$ Division of Education UMAE Hospital de Especialidades \# 14 CMN Adolfo Ruiz Cortines, IMSS, Veracruz, Ver, Mexico

${ }^{4}$ Coordination of planning and institutional liaison OOAD Veracruz Norte, Mexican Social Security Institute, Xalapa, Veracruz, Mexico

\section{Abstract}

Introduction: Acute pancreatitis is a common disease, characterized by inflammation and destruction of the pancreas. There are multiple scales for its assessment within which are the Ranson prognostic scale and the Apache II scale. Our objective is to know the prognostic evaluation between Apache II and Ranson scales in severe acute pancreatitis in the emergency department.

Objective: To know the prognostic evaluation between Apache II and Ranson scale in severe acute pancreatitis, in the emergency department.

Material and methods: A retrospective, cross-sectional, comparative and observational study was carried out in patients older than 18 years with acute pancreatitis in a period of 3 months, the Apache II and Ranson scale were applied; They were classified as: mild and severe. Non-probabilistic samples, for convenience. The severity predictors were compared using the confidence intervals, area under the ROC curve (Receiver Operating Characteristics), significance level of p: $<0.05 \%$.

Results: Mild pancreatitis was higher, Ranson being 39\% mild, 21\% grave, and Apache II scale, 57\% mild, and $43 \%$ grave. The APACHE II scale has a higher sensitivity (65.52) compared to the RANSON scale (43.10). In our environment, it obtained a fairly acceptable value, compared to the Ranson scale.

Conclusion: The APACHE II scale presented a higher sensitivity with respect to the RANSON scale.

Keywords: Apache II scale; Ranson scale; Severe acute pancreatitis

\section{Introduction}

Acute Pancreatitis (AP) is an inflammatory process of the pancreas with variable involvement of other regional tissues or distant organs [1,2]. The Atlanta classification defines AP as mild where organ dysfunction is minimal, usually self-limited and with an uncomplicated and serious recovery which is characterized by multiple organ failure and local complications such as necrosis [3-5]. Mild acute pancreatitis occur in $80 \%$ of cases and severe pancreatitis in the remaining $20 \%$, its annual incidence ranges from 50 to 500 cases per million inhabitants [4-7]. At present, it continues to be a disease characterized by significant morbidity and mortality, with an overall mortality of $5 \%[6,8]$. In Mexico it is the 20th cause of mortality, causing $0.5 \%$ of deaths in the country [6-9]. It is important to detect and staging patients with AP in order to decide who requires an intensive care unit [10]. Therefore, several prognostic scoring systems have emerged which are used to predict the severity of the disease in the first days of diagnosis, among which are Ranson and Apache II [6].

The Ranson scale is important in assessing severity, in which values greater than 3 indicate severity data, although it has the disadvantage of requiring a complete assessment 
at $48 \mathrm{hrs}[6,11,12]$. On the other hand, the Apache scale II can be calculated at any time during the BP, even during the first hours, where a count greater than or equal to 8 points indicates severe pancreatitis, it has the advantage of being the most accurate predictor with acceptable rates of sensitivity and specificity $[3,10,13,14]$. Both multifactorial clinical scoring systems have been useful in assessing the severity of acute pancreatitis, although the disadvantages of these scoring scales are that they are not designed to predict possible complications of the disease [6]. Therefore, all patients admitted to the emergency department with a diagnosis of acute pancreatitis will have to be evaluated with the Apache II and Ranson prognostic scales with the intention of determining those who potentially develop a severe acute pancreatitis picture. In Mexico the evaluations of the prognostic scales or parameters for acute pancreatitis have been little studied, which is why it is of vital importance to evaluate and review them, fifteen therefore, the objective of this present is to know the prognostic evaluation between Apache II and Ranson scale in severe acute pancreatitis, in the emergency department

\section{Material and Methods}

Retrospective, cross-sectional, comparative and observational design, in 60 patients older than 18 years of age, with a clinical picture suggestive of acute pancreatitis in the adult emergency department in a period of 3 months, demographic variables and Apache II scale and scale of Ranson. Acute pancreatitis cases were classified as: Mild and severe according to the Atlanta consensus of 1992; The general objective was to determine the prognostic efficacy between the Apache II scale and the Ranson scale in severe acute pancreatitis and as specific objectives to determine the degree of mortality and determine complications according to gender. Non-probabilistic samples, for convenience. The study was carried out with prior authorization from the local ethics and research committee of the medical unit. Statistical analysis: Prognostic scales were used through their sensitivity, specificity and prediction values, the predictive capacity of each indicator was estimated by the area under the ROC curve (Receiver Operating Characteristics). The severity predictors were compared using the confidence intervals of their areas under the ROC curve, significance level of $0.05 \%$, statistical analysis in SPSS v 20 and Epidat 3.1

\section{Results}

It was possible to determine that there is a higher prevalence of this pathology in female patients with 60\%, (n:36), Figure 1, having a mean age of $51.58 \%$, Table 1 . The pancreatitis distribution was higher in pancreatitis mild, obtaining by means of the Ranson scale $39 \%$ of the pancreatitis were mild and $21 \%$ severe and with the measurement made with the Apache II scale 57\% were mild and $43 \%$ were classified as severe Table 2 . Each prognostic score has its own sensitivity, Specificity and feasibility, in the present study it was found that the Apache II Scale presents greater sensitivity (65.52) compared to the RANSON scale (43.10), with a low specificity of 26.0 , and a PPV of $97 \%$ Table 3a\&3b. The comparison of scales is specified in Figure 2. The area of the ROC curve for the Apache scale was 0.793 , Figure 3.

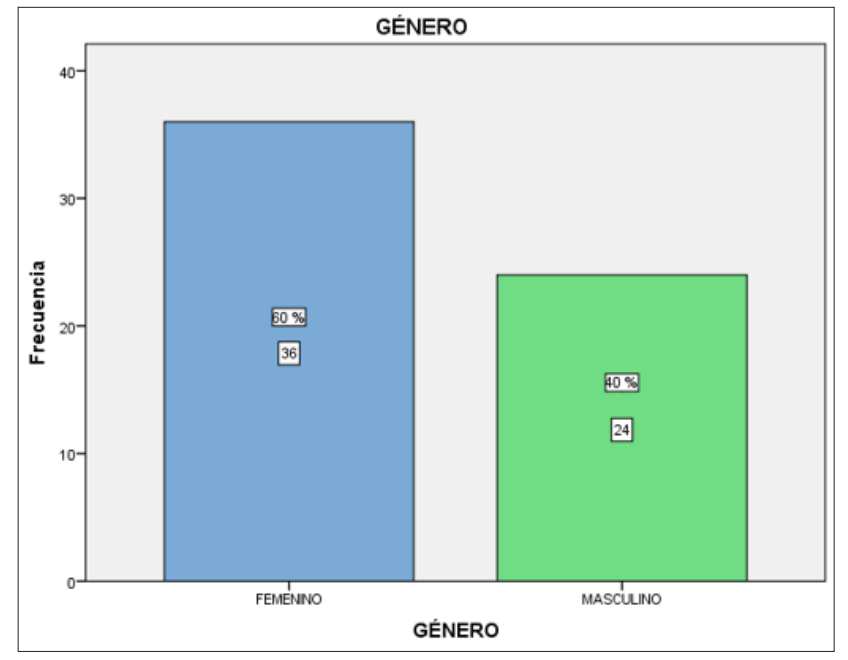

Figure 1: Distribution by gender.

Table 1: Age distribution by gender.

\begin{tabular}{|c|c|c|c|c|c|c|}
\hline \multicolumn{7}{|c|}{ Age in Years by Gender } \\
\hline Gender & Half & n & DS & Minimum & Maximum & Rank \\
\hline Female & 50.08 & 36 & 14.02 & 21 & 83 & 62 \\
\hline Male & 53.83 & 24 & 12.82 & 26 & 77 & 51 \\
\hline Total & 51.58 & 60 & 13.57 & 21 & 83 & 62 \\
\hline
\end{tabular}

Table 2: Scale comparison.

\begin{tabular}{|c|c|c|c|c|c|c|}
\hline \multicolumn{7}{|c|}{ Apache Severity Assessment } \\
\hline & & Mild & $\begin{array}{c}\text { Seri- } \\
\text { ous }\end{array}$ & Total & $\mathrm{p}$ & \multirow{7}{*}{0.95} \\
\hline \multirow{6}{*}{$\begin{array}{c}\text { Ranson } \\
\text { Severity } \\
\text { Assessment }\end{array}$} & \multirow{2}{*}{ Mild } & Frequency & 17 & 22 & 39 & \\
\hline & & $(\%)$ & 28.3 & 36.7 & 65 & \\
\hline & \multirow{2}{*}{ Serious } & Frequency & 9 & 12 & 21 & \\
\hline & & $(\%)$ & 15 & 20 & 35 & \\
\hline & \multirow{2}{*}{ Total } & Frequency & 26 & 34 & 60 & \\
\hline & & $(\%)$ & 43.3 & 56.7 & 100 & \\
\hline
\end{tabular}

Table 3a: Sensitivity, specificity, V.P.P, V.P.N, ranson prevalence.

\begin{tabular}{|c|c|c|c|}
\hline & Value & \multicolumn{2}{|c|}{ I.C 95\% } \\
\hline Sensitivity & 43.10 & 25.50 & 56.71 \\
\hline Specificity & 50.00 & 0.00 & 100 \\
\hline V.P.P & 96.15 & 86.84 & 100 \\
\hline V.P.N & 2.94 & 0.00 & 10.09 \\
\hline Prevalence & 96.67 & 91.29 & 100 \\
\hline
\end{tabular}


Table 3b: Sensitivity, specificity, V.P.P, V.P.N, apache II prevalence.

\begin{tabular}{|c|c|c|c|}
\hline & Value & I.C 95\% & \\
\hline Sensitivity & 65.52 & 52.42 & 78.61 \\
\hline Specificity & 26.00 & 0.00 & 100 \\
\hline V.P.P & 97.44 & 91.19 & 100 \\
\hline V.P.N & 4.76 & 0.00 & 16.25 \\
\hline Prevalence & 96.67 & 91.29 & 100 \\
\hline
\end{tabular}

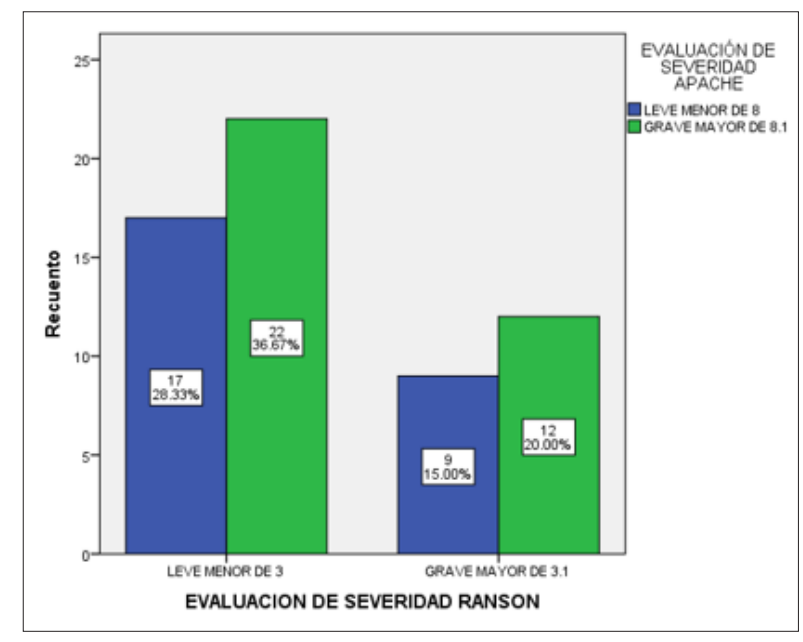

Figure 2: Comparison of scales.

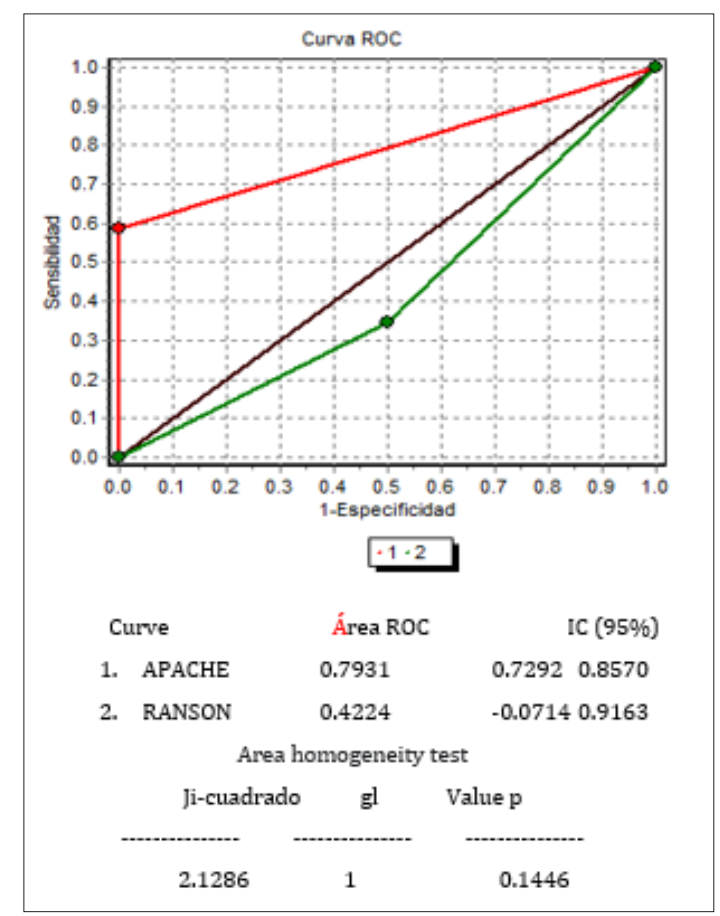

Figure 3: Comparison of scales.

\section{Discussion}

The immense knowledge harbored over the years since the Atlanta Symposium of 1992 to date, the basic concepts and the forecast scales have been modified with scientific advance, however until now and of course Mexico belongs to the conglomerate In still developing countries and with various logistical problems, it is common not to have most of the serological markers and radiology studies, with which, at a global level, the evolution and severity of this can be evaluated and predicted early suffering. For this reason, various scales are used that are accessible, inexpensive, and easy to perform in the emergency and surgical departments of the country, with good sensitivity and specificity. Predicting the clinical course that pancreatitis symptoms will follow has led to the search for simple, easy-to-obtain, cheap, accessible and highly effective indicators [9] that, applied early, allow the identification of severe conditions for vigorous therapeutic intervention. In this study, 60 patients were included, with $60 \%$ of the patients being female and $40 \%$ male, being comparable to that found by Reibán at the Vicente Moscoso Hospital in which a higher prevalence was found in women in a $68 \%$ and in men $31.02 \% 10$. Likewise, both Ranson and Apache II scales were applied to the universe of 60 patients, so that according to the parameters obtained at admission, the degree of severity of pancreatitis (Mild-Severe) could be determined, establishing as a cut-off point for the Ranson criteria (Mild, less than 3 points and Major of 3.1), and for the Apache II scale (Minor less than 8 points and Major of 8.1). Where a significant difference was found in the ROC curve for the Ranson scale of 0.42 below the curve, and 0.79 for the Apache II scale. Data that are similar to those found by Y. Surco et al. Who describe a statistically significant difference between Ranson with an area under the ROC curve for Ranson of 0.41 and an area under the ROC curve for Apache II of 0.74 [10]. Ranson's criteria, Imrie's criteria and other multifactorial parameters can be used to detect mild forms ( $<3$ points) but they are "not useful" to predict severity; In addition, 48 hours are required to complete all the criteria, so they are not useful for an initial assessment, data from are based on the obtained sensitivity of 43.10, with a specificity of 50.00, with a PPV of 36.15, and a NPV of 76.36. Contrary to the Apache II scale, it improves the precision to establish the prognosis, with a positive predictive value (PPV) of 97.4, a NPV of 4.76 , a sensitivity of 65.52 , and a specificity of 26.0 admission and does not predict local complications, however, it has the main advantage that its efficacy at $24 \mathrm{~h}$ is similar to that of traditional systems at $48 \mathrm{~h}$. Likewise and although the prediction of gravity is still a valuable concept. The identification of early markers of persistent organ failure is important because it is a concern to predict the actual factors of severity pancreatic necrosis and/or organ failure). A recent example of this is the measurement of angiopoietin 2, a marker of vascular leak syndrome, in the prediction of persistent organ failure, [11] which is not available in our setting, so indirect markers are still used inflammatory that although nonspecific support the clinical diagnostic criteria.

The sensitivity of serum pancreatic enzymes in the diagnosis of acute pancreatitis is different and basically depends on the speed with which each one of them clears from the circulation. Based 
on this clarification of circulating enzymes, the cut-off point for your serum levels for the diagnosis of acute pancreatitis cannot be fixed (usually 2-3 times above the upper limit of normal), but rather variable, depending on the time elapsed since the onset of the disease [12]. But they are very nonspecific to consider them as severity parameters, but nevertheless they are useful in our hospital environment, for which the amylase levels obtained in the first $24 \mathrm{hrs}$ of admission were collected, taking values above 30 a $118 \mathrm{U} / \mathrm{L}$ of amylase, finding a higher sensitivity, a specificity of $60 \%$, as well as a PPV of 66.5. Data similar to those obtained in the study obtained by Dr. Carlos Antonio Gutierrez at the Calixto García University Hospital in Cuba, who determined a sensitivity of $82 \%$, a specificity of $88 \%$ with a PPV of $65 \%$ [13]. Taking into account that to amylase: it can be elevated in a myriad of pathologies, which include obstruction of the pancreatic duct, alcohol consumption, mumps, kidney disease and peptic ulcers, but based on The results found in our study corroborate the fact that it is a good indicator parameter of the severity of the disease if it is accompanied by a correct clinical suspicion and we add it to the laboratory and Cabinet tools [14].

\section{Conclusion}

Acute pancreatitis is more prevalent in female patients. It was found that based on the results obtained, the distribution of pancreatitis was greater in mild pancreatitis. Each prognostic Score has its own sensitivity, specificity and feasibility, in the present study it was found that the APACHE II Scale presents greater sensitivity (65.52) compared to the RANSON scale (43.10), low specificity of (26.0), however with a PPV of 97\%. Therefore, it is translated that this scale, although in contrast to the international literature, places it with a low positive predictive value, in our environment it obtained a fairly acceptable value, placing it as the best scale compared to Ranson. The Ranson scale, even with its limitations because we cannot determine it before 48 hours, can continue to be used in our hospital as a prognostic factor of severity, taking into account the low specificity (50.00), with a sensitivity of (43.10), and a high NPV of (76.36), PPV of (36.15) so the use of said score is left to medical consideration.

\section{References}

1. Duffy B, Mijares J, Ortega L, Perez H, Cárdenas L (2003) Comparison of two multiple parameter prognostic systems (APACHE II and Ranson) in acute pancreatitis. General Surgeon 25(2): 112-118.

2. Bradley EL (1993) A clinically based classification system for acute pancreatitis. Summary of the international symposium on acute pancreatitis, atlanta, Ga, september 11 through 13, 1992. Arch Surg 128(5): 586-590.

3. Pascual M, Martinez J (2012) Acute pancreatitis. Medicine: Accredited Continuing Medical Training Program 11: 457-464.

4. Ledesma JP, Arias J (2009) Acute pancreatitis. Med Int Mex 25(4): 285294.

5. Skipworth J, Pereira S, James RA (2008) Acute pancreatitis. Curr Opin Crit Care 14(2): 172-178.

6. Gonzalez F, Garcia K, Alvarez F (2014) Validation of the BISAP, APACHE II and RANSON scales to predict organ failure and complications in acute pancreatitis. Rev Med MD 4.5(2): 66-72.

7. Carrol JK, Brian H, Teresa G (2007) Acute pancreatitis: Diagnosis, prognosis, and treatment. Am Fam Physician 75(10): 1513-1520.

8. Sandra B, Olaf J, Marc G, Thomas L, Paul F, et al. (2012) Treatment of necrotizing pancreatitis. Clin Gastroenteral Hepatol 10(11): 1190-1201.

9. Taylor SL, Daniel L, Kent D, Mary M, Larry R (2005) A comparison of the Ranson, Glasgow, and APACHE II scoring systems to a multiple organ system score in predicting patient outcome in pancreatitis. Am J Surg 189(2): 219-222.

10. Remes M, Amaya T, Bosques FJ, Guzmán MT, Marín López E, et al. (2007) Clinical guidelines for the diagnosis and treatment of acute pancreatitis epidemiology, etiology, diagnosis and treatment. Rev Gastroenterol Mex 72(4): 387-389.

11. De Bernardinis M, Violi V, Roncoroni L, Boselli AS, Giunta A, et al. (1999) Discriminating power and information content of Ranson's prognostic signs in acute pancreatitis: A meta-analytic study. Critical Care Medicine 27(10): 2272-2283.

12. Papachristou GI, Gilles C, Arun Sharma, Dhiraj Yadav, David C (2007) Risk and markers of severe acute pancreatitis. Gastroenterology Clinics of North America 36(2): 277-296.

13. Meijide H (1999) Apache II. Predictors of severity in acute pancreatitis. Internal Medicine.

14. Martín G, Cecilia C, Analía P, Hui J, Hugo P, et al. (2019) Update on the initial management of acute pancreatitis. Acta Gastroenterol Latinoam 49(4): 307-323. 